\title{
(Ga,Mn)As-Based Magnetic Tunnel Junctions under Electric Field from First Principles
}

\author{
M. LUO ${ }^{a, *, \dagger}$ AND Y.H. SHEN ${ }^{b}$ \\ ${ }^{a}$ Department of Electronic Engineering, Shanghai JianQiao University, Shanghai 201306, People's Republic of China \\ ${ }^{b}$ Key Laboratory of Polar Materials and Devices, East China Normal University, Shanghai 200241, \\ People's Republic of China
}

(Received April 12, 2016; in final form November 15, 2016)

\begin{abstract}
Diluted magnetic semiconductors composed in magnetic tunnel junctions have potential applications in spintronics but the development has not been fast. The main difficulty is how to control the magnetism of diluted magnetic semiconductors. For our model semiconductor magnetic tunnel junctions, (Ga,Mn)As/GaAs/(Ga,Mn)As, we found that the magnetic coupling between the transition metal ions in each diluted magnetic semiconductor electrode of such semiconductor magnetic tunnel junctions can be switched from ferromagnetic to antiferromagnetic by the external electric field. The phenomenon suggest a possible avenue for the application of semiconductor magnetic tunnel junctions.
\end{abstract}

DOI: 10.12693/APhysPolA.130.1385

PACS/topics: 73.61.Ey, 75.30.Et, 75.50.Pp

\section{Introduction}

Electric-field effect on magnetism, first demonstrated in ferromagnetic semiconductors $[1,2]$, is now being extended to other material systems including $3 d$ transition metals [3, 4] attracting much attention not only from the physics point of view but also from the applications point of view. Recently, electrical switching of magnetization direction was realized for a free layer of magnetic tunnel junctions (MTJs) through the change of magnetic anisotropy by applying nanosecond electricfield pulses [5, 6]. Most studied MTJs are based on ferromagnetic (FM) metal electrodes and are assembled as ferromagnet/insulator/ferromagnet. Semiconductor MTJs (SMTJs) are composed of two diluted magnetic semiconductor (DMS) electrodes and a semiconductor barrier film assembled as a DMS/semiconductor/DMS junction, and are expected to become important in future spintronics devices due to the fact [7] that high-quality SMTJs can be readily prepared and integrated into other devices and an important material property such as energy gap can be easily modified. Many SMTJs investigated so far are based on GaAs [7], SbTe [8], GaN [9], and $\mathrm{ZnO}[10]$, but their spin-dependent transport properties are not well understood. The applications of SMTJs have been difficult because the tunnel resistance shows a sizeable variation due to the uncertainty in the magnetism of the DMS electrodes. For instance, in the most representative DMS system, GaMnAs, reliable ferromagnetism resulting from the substitutional doping of $\mathrm{Mn}-\mathrm{Ga}$ has been widely observed [11-14]. To enable the practical

\footnotetext{
* corresponding author; e-mail: mluo@gench.edu.cn

${ }^{\dagger}$ mailing address: NO.1111, Huchenghuan Road, Pudong New District, Shanghai, China
}

application of SMTJs, it is crucial to develop an effective way of controlling the magnetic order in the DMS components.

\section{Calculation details}

In the present work, we propose the application of external electric field $\varepsilon$ across a SMTJ as an effective method to control the magnetism of its DMS electrodes because the applied electric field can induce electron transfer from one DMS electrode to the other DMS electrode and because the magnetic properties of the DMS electrodes depend on the number of electrons present in their band gap states. The use of external electric field is known to strongly affect the electronic properties of other materials [15-17]. To verify the feasibility of this proposal, we carry out first principles density functional theory (DFT) calculations for model SMTJs, (Ga,Mn)As/GaAs/(Ga,Mn)As under electric field. All our DFT electronic calculations were performed using the projector augmented wave method [18] encoded in the Vienna ab initio simulation package (VASP) with the generalized gradient approximation [19]. In the VASP the planar dipole layer method [20] is employed to include electric field in the Hamiltonian and has been successfully applied in several studies [21-23]. In this method an artificial dipole sheet is placed in the middle of the vacuum part, and the electrostatic potential decreases along the direction of the electric field.

To simulate the SMTJs, we construct a slab consisting of 10 layers of GaAs parallel to the (110) surface plus a vacuum layer with thickness of $10 \AA$, with 225 supercell, as shown in Fig. 1. For convenience, we label these layers as $T_{n}$ and $B_{n}(n=1-3)$ such that $T_{1}$ and $B_{1}$ refer to the surface GaAs layers on the top and bottom of the junctions, respectively. To make a SMTJ, we introduce two $\mathrm{Mn}^{3+}$ ions in each of the $T_{n}$ and $B_{n}$ layers, which 
will be referred to as the $\left(T_{n}, B_{n}\right)$ model. Here, it is crucial to make sure both top and bottom ( $\mathrm{Ga}, \mathrm{Mn})$ As layers are FM. In the Mn-doped GaAs system, the FM interaction is dependent on the distance between two Mn atoms due to their short-range interactions [24, 25]. Thus, in each $(\mathrm{Ga}, \mathrm{Mn})$ As layer containing two $\mathrm{Mn}^{3+}$ dopants, we arrange them in the nearest-neighbor pairs. This corresponds to the dopant concentration of $5.0 \%$ (i.e., four $\mathrm{Mn}^{3+}$ ions in the $80 \mathrm{Ga}^{3+}$ sites). In the absence of applied electric field, the $\mathrm{Mn}^{3+}\left(d^{4}\right)$ ions at the tetrahedral sites exist as high-spin ions with electron configurations $(e \uparrow)^{2}\left(t_{2} \uparrow\right)^{2}$.

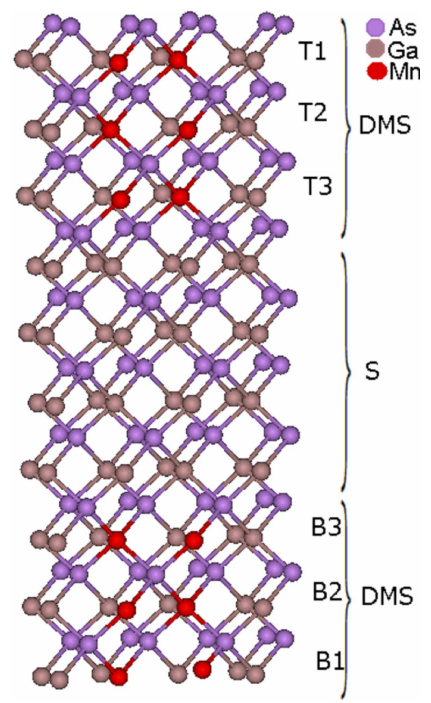

Fig. 1. Model SMTJs based on the slab of 10 layers of GaAs parallel to the (110) surface of GaAs. Only the atoms contained in a unit cell of the $2 \times 2 \times 5$ supercell structure are presented.

\section{Results and discussion}

For the (Ga,Mn)As/GaAs/(Ga,Mn)As junction, we mainly considered the $T_{3}, B_{3}$ model. As found in the previous theoretical and experimental studies [26], our calculations show that the $\mathrm{Mn}^{3+}$ ions in each DMS electrodes favor ferromagnetic (FM) coupling. Magnetic coupling between the two DMS electrodes is observed in our calculations. To induce electron transfer from one DMS electrode to the other and consequently alter the magnetism of each DMS electrode, we apply an external electric field across the SMTJs from the bottom to the top. For the $\left(T_{3}, B_{3}\right)$ model, Fig. 2 shows the magnetic coupling between the $\mathrm{Mn}^{3+}$ ions in the DMS electrode (Ga,Mn)As is intrinsically FM. We also calculated that the $\left(T_{2}, B_{2}\right)$ and $\left(T_{1}, B_{1}\right)$ models show FM coupling between the $\mathrm{Mn}^{3+}$ ions under zero field. The energy differences between FM and AFM states are -27 and $16 \mathrm{meV}$, respectively, which are much smaller than that of $\left(T_{3}, B_{3}\right)$ model $(-58 \mathrm{meV})$. These results indicate that the FM coupling becomes much more weaker, that because of electronic and magnetic structures of this DMS is greatly affected by the surfaces [27]. Thus, we study the electronic properties of the $\left(T_{3}, B_{3}\right)$ model under the electric field. When $\varepsilon=0$, as anticipated, the (Ga,Mn)As $/ \mathrm{GaAs} /(\mathrm{Ga}, \mathrm{Mn})$ As junction is FM. But when $\varepsilon>0$, the (Ga,Mn)As/GaAs/(Ga,Mn)As junction shows a change. The interaction between adjacent $\mathrm{Mn}$ ions in the DMS switch from FM to AFM at $\varepsilon=0.2 \mathrm{~V} / \AA$. Namely, the Mn magnetic moment in the junctions changes immediately $\left(4.05 \mu_{\mathrm{B}}\right.$ to $\left.0.22 \mu_{\mathrm{B}}\right)$ upon the application of electric field, whereas an electric field beyond a critical value is necessary for the electron transfer in the (Ga,Mn)As/GaAs/(Ga,Mn)As junction. Therefore, the magnetic ordering in the (Ga,Mn)As $/ \mathrm{GaAs} /(\mathrm{Ga}, \mathrm{Mn}) \mathrm{As}$ junction changes from FM to AFM by external electric field, Fig. 2.

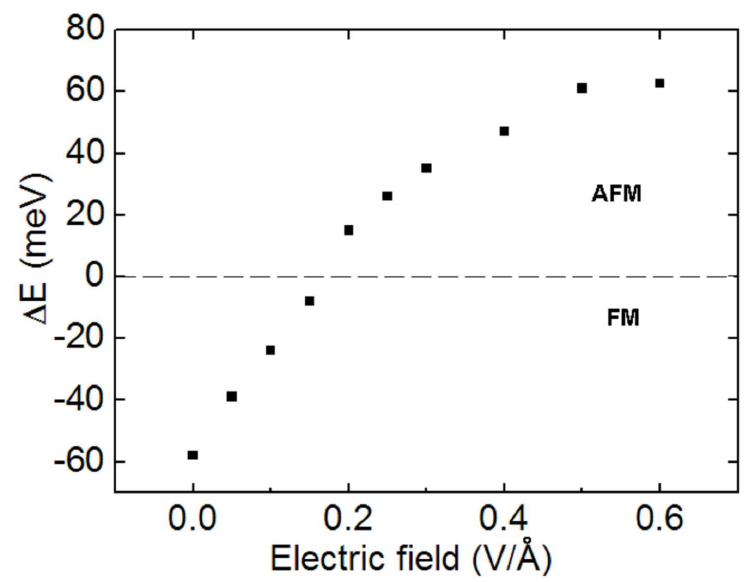

Fig. 2. The energy difference between the AFM and FM states of the (Ga,Mn)As/GaAs/(Ga,Mn)As junction calculated by using the $\left(T_{3}, B_{3}\right)$ model.

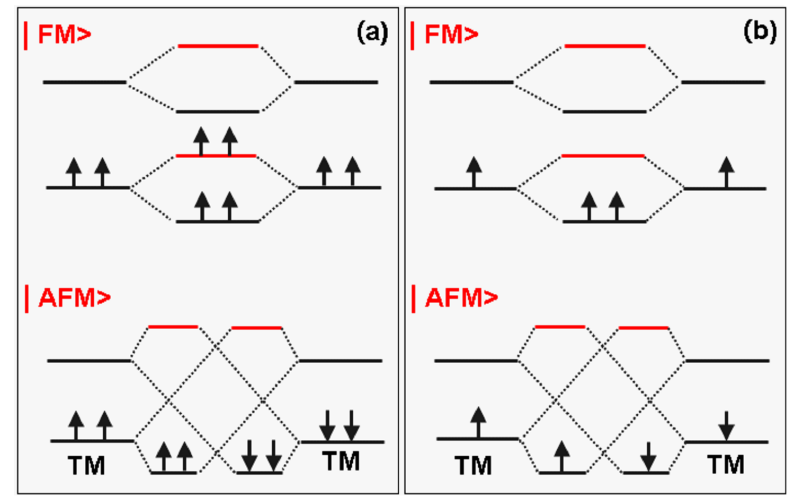

Fig. 3. Interactions between adjacent $\mathrm{TM}$ ions in a DMS: (a) the FM and AFM interactions for the TM ions with completely filled $d$ states and (b) those for the TM ions with incompletely filled $d$ states.

The FM or AFM coupling between the doped transition-metal (TM) ions in a DMS electrode is explained by considering the orbital interactions between their $d$ states $[24,25]$. For the FM arrangement between adjacent TM ions (Fig. 3a), each completely filled state 
(either up- or down-spin state) of one TM ion interacts with the corresponding state of the other TM ion so that the resulting bonding and antibonding states are both filled, hence leading to no energy gain. For the AFM arrangement between adjacent TM ions, however, there occur interactions in which a completely filled state of one TM ion interacts with a completely empty state of the other TM ion. In this case only the resulting bonding states are filled, hence leading to energy lowering (Fig. 3a). Thus, the preferred magnetic coupling between adjacent TM ions should be AFM. If holes (electrons) are introduced into the TM ions (Fig. 3b) the $d$ states of the TM ions are partially filled so that the preferred magnetic coupling between the TM ions becomes FM because the antibonding (bonding) states derived from the interaction become empty (filled) (Fig. 3b).

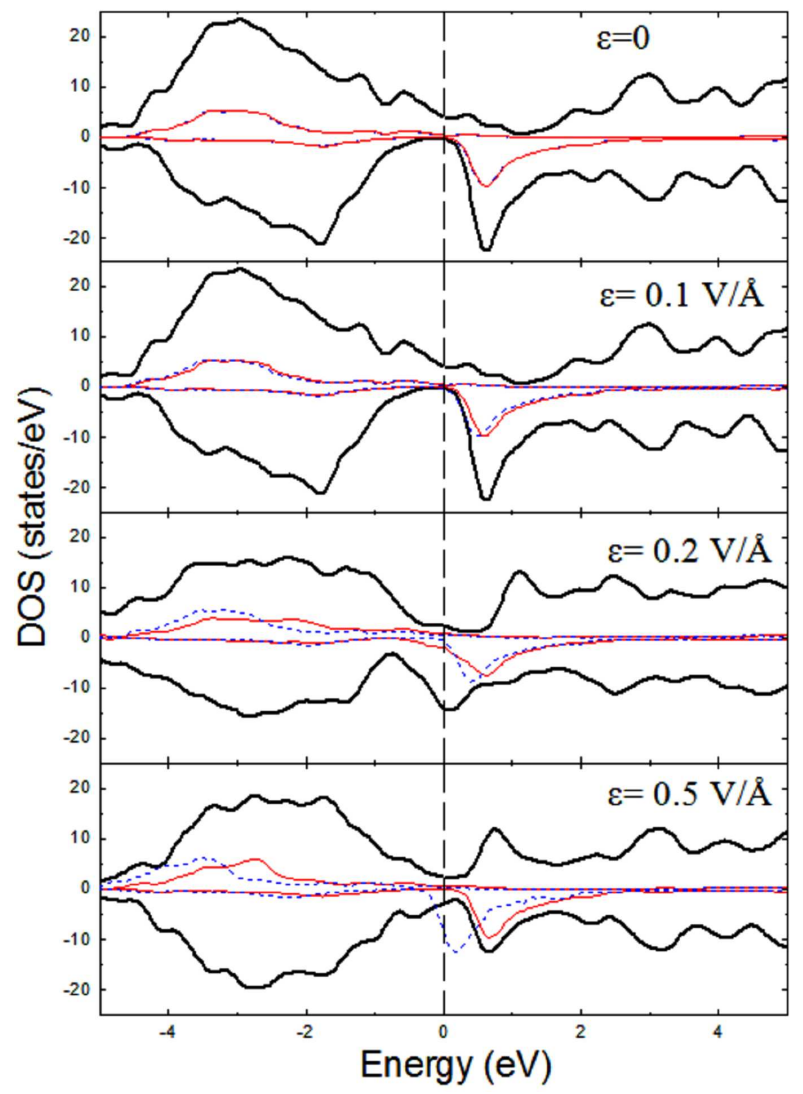

Fig. 4. DOS plots of the FM states calculated for the $\left(T_{3}, B_{3}\right)$ model of $(\mathrm{Ga}, \mathrm{Mn}) \mathrm{As} / \mathrm{GaAs} /(\mathrm{Ga}, \mathrm{Mn})$ As. The red solid and blue dotted lines represent the partial DOS plots for the Mn atoms of the top and bottom DMS electrodes, respectively, while the black thick line represents those for GaAs atoms.

Our SMTJs have no external carrier doping but the calculated magnetic moments of their TM ions show a behavior expected for carrier-doped TM ions. This is so because the applied electric field induces electron transfer from one DMS electrode to the other. The density of states (DOS) plots calculated as a function of $\varepsilon$ for the (Ga,Mn)As/GaAs/ (Ga,Mn)As junctions are summarized in Fig. 4. For the (Ga,Mn)As/GaAs/(Ga,Mn)As at $\varepsilon=0$, the $\mathrm{Mn}^{3+}$ up-spin $t_{2}$ states are partially filled, hence leading to a FM coupling between the $\mathrm{Mn}^{3+}$ ions. The applied electric field raises the TM $d$ states of the top DMS electrode while lowering those of the bottom DMS electrode. This effect becomes stronger with increasing $\varepsilon$ thereby enhancing the electron transfer from the top to the bottom DMS electrode. For the (Ga,Mn)As/GaAs/(Ga,Mn)As junction, there is no energy gap to close because the Mn up-spin $t_{2}$ states are partially filled. As a result, electrons are immediately transferred from one DMS electrode to the other upon the application of electric field. With strong enough electric field, the Mn up-spin $t_{2}$ states in the bottom electrode become fully filled with a small amount of electrons left on the Mn up-spin $t_{2}$ states in the top electrode so that the preferred magnetic coupling between the Mn atoms in each electrode is changed from FM to AFM.

\section{Conclusion}

In summary, we have investigated the (Ga,Mn)As GaAs/(Ga,Mn)As SMTJs by electric field effect, we find that the SMTJs are predicted to undergo a magnetic switching under electric field due to the electron transfer from one DMS electrode to another. This phenomenon suggests a useful method to control the magnetism of DMSs and hence extend the functionality of SMTJs.

\section{Acknowledgments}

We thank the Supercomputer Center of ECNU for using the Dawn 5000A supercomputer. The work is supported by the research programs of Shanghai (Z31004.15.03).

\section{References}

[1] H. Ohno, D. Chiba, F. Matsukura, T. Omiya, E. Abe, T. Dietl, Y. Ohno, K. Ohtani, Nature 408, 944 (2000).

[2] D. Chiba, M. Yamanouchi, F. Matsukura, H. Ohno, Science 301, 943 (2003).

[3] D. Chiba, M. Sawicki, Y. Nishitani, Y. Nakatani, F. Matsukura, H. Ohno, Nature 455, 515 (2008).

[4] M. Endo, S. Kanai, S. Ikeda, F. Matsukura, H. Ohno, Appl. Phys. Lett. 96, 212503 (2010).

[5] Y. Shiota, S. Miwa, T. Nozaki, F. Bonell, N. Mizuochi, T. Shinjo, H. Kubota, S. Yuasa, Y. Suzuki, Appl. Phys. Lett. 101, (2012) 102406.

[6] S. Kanai, M. Yamanouchi, S. Ikeda, Y. Nakatani, F. Matsukura, H. Ohno, Appl. Phys. Lett. 101 (2012) 122403.

[7] H. Saito, S. Yuasa, K. Ando, Phys. Rev. Lett. 95, 086604 (2005).

[8] Z. Zhou, Y. Chien, C. Uher, Appl. Phys. Lett. 89, 232501 (2006). 
[9] M. Kim, Y. Zhou, M. Funakoshi, S. Emura, Appl. Phys. Lett. 89, 232511 (2006).

[10] C. Song, X. Liu, F. Zeng, F. Pan, Appl. Phys. Lett. 91, 042106 (2007).

[11] T. Dietl, H. Ohno, F. Matsukura, J. Cibert, D. Ferrand, Science 287, 1019 (2000).

[12] K. Ueda, H. Tabata, T. Kawai, Appl. Phys. Lett. 79, 988 (2001).

[13] A. Behan, A. Mokhtari, H. Blythe, D. Score, X. Xu, J. Neal, A. Fox, G. Gehring, Phys. Rev. Lett. 100 , 047206 (2008).

[14] M. Sawicki, D. Chiba, A. Korbecka, Y. Nishitani, J.A. Majewski, F. Matsukura, T. Dietl, H. Ohno, Nat. Phys. 6, 22 (2010).

[15] M. Weisheit, S. Fahler, A. Marty, Y. Souche, C. Poinsignon, D. Givord, Science 315, 349 (2007).

[16] E. Kan, Z. Li, J. Yang, J. Hou, Appl. Phys. Lett. 91, 243116 (2007)

[17] C. Duan, J. Velev, R. Sabirianov, Z. Zhu, J. Chu, S. Jaswal, E. Tsymbal, Phys. Rev. Lett. 101, 137201 (2008).
[18] P. Blöchl, Phys. Rev. B 50, 17953 (1994); G. Kresse, J. Furthmuller, Phys. Rev. B 54, 11169 (1996).

[19] D. Ceperley, B. Alder, Phys. Rev. Lett. 45, 566 (1980).

[20] J. Neugebauer, M. Scheffler, Phys. Rev. B 46, 16067 (1992).

[21] R. Ramazashvili, Phys. Rev. Lett. 105, 216404 (2010).

[22] Y. Guo, W. Guo, C. Chen, Appl. Phys. Lett. 92, 243101 (2008).

[23] G. Lee, K. Cho, Phys. Rev. B 79, 165440 (2009).

[24] A. Walsh, J. Da Silva, S. Wei, Phys. Rev. Lett. 100, 256401 (2008).

[25] E. Kan, L. Yuan, J. Yang, J. Appl. Phys. 102 , 033915 (2007).

[26] M. Luo, Z. Tang, J. Zheng, Z.Q. Zhu, J.H. Chu, J. Appl. Phys. 108, 053703 (2010).

[27] Q. Wang, Q. Sun, P. Jena, Y. Kawazoe, Phys. Rev. Lett. 93, 155501 (2004). 